\title{
La traduction des ouvrages des ingénieurs : stratégies d'auteurs, pratiques de libraires et volonté des princes (1600-1750)
}

Translating engineers' books: authors' strategies, booksellers' practices and princely politics (1600-1750)

\section{Michèle Virol}

\section{(2) OpenEdition}

\section{Journals}

Édition électronique

URL : http://journals.openedition.org/artefact/417

DOI : $10.4000 /$ artefact. 417

ISSN : 2606-9245

Éditeur :

Association Artefact. Techniques histoire et sciences humaines, Presses universitaires du Midi

Édition imprimée

Date de publication : 1 octobre 2016

Pagination : 181-195

ISBN : 978-2-7535-5174-9

ISSN : 2273-0753

\section{Référence électronique}

Michèle Virol, «La traduction des ouvrages des ingénieurs : stratégies d'auteurs, pratiques de libraires et volonté des princes (1600-1750) », Artefact [En ligne], 4 | 2016, mis en ligne le 07 juillet 2017, consulté le 02 mai 2019. URL : http://journals.openedition.org/artefact/417 ; DOI : 10.4000/ artefact. 417 


\section{La traduction des ouvrages des ingénieurs : stratégies d'auteurs, pratiques de libraires et volonté des princes $(1600-1750)$}

Michèle VIROL

\section{Résumé}

Les ingénieurs militaires ont fait connaître leurs savoirs en rédigeant des traités précis sur les techniques qu'ils maitrisaient. Dans un contexte de guerre, ces traités ont été parfois édités et traduits, donnant à leurs auteurs une reconnaissance souvent européenne. Soit qu'il s'agisse d'une politique volontaire de l'auteur, de la demande d'un prince ou d'une stratégie d'imprimeurs-libraires voulant profiter de la demande d'un lectorat curieux, de connaître les secrets des ingénieurs réputés, les traductions sont nombreuses, notamment celles des ouvrages français.

Mots-clés : fortification, imprimeur libraire, ingénieur, mobilité, traduction.

\section{Abstract. Translating engineers' books: authors' strategies, booksellers' practices and princely politics (1600-1750)}

Military engineers spread their knowledge by writing treatises on technologies they were mastering. These works often were manuscript but they seldom were published and translated, confering fame to their authors. These translations were numerous, especially in French. They either followed authors' aims to publish, or answered the requests of monarchs or matched

*. Michèle Virol, professeur d'histoire moderne à l'université de Normandie-Rouen, travaille sur la guerre, le politique et les techniques au $\mathrm{XVII}^{\mathrm{e}}$ siècle, en privilégiant les ingénieurs, leurs réalisations et leurs écrits. Elle a récemment publié Au cour des querelles politiques et religieuses sous Louis XIV. Vincent Ragot de Beaumont (Rouen, PURH, 2013) et Louis XIV et Vauban. Édition critique de leur correspondance (Seyssel, Champ Vallon, à paraître fin 2016). Contact [m_virol@yahoo.fr]. 
printers-booksellers' strategies who wanted to benefit from an interested audience, in a context of warship.

Keywords : engineer, fortification, mobility, printer-bookseller, translation.

Les multiples écrits des ingénieurs concernent plusieurs sujets : armée, attaque et défense des places, artillerie et balistique, architecture militaire et civile, construction de bateaux, hydraulique, ponts et routes, cartographie, mathématiques appliquées au toisé et à l'arpentage, mais aussi récits de siège ou descriptions de villes et de ports. Au $\mathrm{XVII}^{\mathrm{e}}$ siècle et dans la première moitié du xvIII $^{\mathrm{e}}$ siècle, la profession d'ingénieur est en pleine mutation, avec une spécialisation qui s'affirme progressivement jusqu'au milieu du xvIII ${ }^{\mathrm{e}}$ siècle. Certains ont un brevet d'ingénieur et servent dans l'armée de terre ou dans la marine; d'autres sont ingénieurs volontaires, engagés pour une campagne ou pour certains travaux; d'autres enfin sont ingénieurs civils, effectuant des travaux d'architecture civile, de voirie, d'ouvrages d'art ou de cartographie. Rares sont ceux qui maîtrisent la totalité des compétences possibles et peu nombreux ceux qui ont laissé des écrits, souvent manuscrits, qui ont parfois circulé sous forme de copies ou d'extraits. Pour ceux qui ont été édités, le rôle joué par les libraires qui s'orientent vers une spécialisation, est essentiel ${ }^{1}$. Quant aux ingénieurs militaires dont il est question dans cet article, ils se chargent des traductions ${ }^{2}$ en fonction de la demande liée à l'actualité des guerres, à la curiosité des princes ou à l'attrait pour les techniques de l'ennemi.
Les ouvrages de fortifications étant souvent secrets, la demande peut être forte au moment des conflits et les éditions pirates plus nombreuses. Des ouvrages peuvent servir de manuels d'enseignement dans le cadre du préceptorat nobiliaire, mais aussi dans les collèges jésuites et oratoriens où l'enseignement de la fortification vient compléter celui des mathématiques. Quand les académies et les écoles d'ingénieurs se développent à la fin du $\mathrm{XvII}^{\mathrm{e}}$ siècle, mais surtout à partir des années 1740, des ouvrages sont réédités et traduits, mais surtout compilés dans des recueils éclectiques.

De cette multiplicité de situations se dégagent les grandes lignes qui permettent de distinguer dans les traductions, donc dans la décision de permettre l'accès du texte à un lectorat étranger, des stratégies personnelles d'auteurs soutenues par des politiques de traduction de libraires imprimeurs ${ }^{3}$. Quant aux langues utilisées pour ces traductions, elles posent plusieurs questions relatives au maintien du latin au $\mathrm{XVII}^{\mathrm{e}}$ siècle et à l'usage d'une langue vernaculaire commune aux ingénieurs.

Les ingénieurs des $\mathrm{XVII}^{\mathrm{e}}$ et $\mathrm{XVIII}{ }^{\mathrm{e}}$ siècles sont très mobiles en raison des nombreux conflits qui leur permettent de changer d'employeurs, des politiques religieuses (la révocation de l'édit de Nantes en 1685) ou dynastiques (la diaspora des partisans de Jacques II Stuart, les Jacobites) ou de carrières contrariées. 
La constitution, au xvII ${ }^{\mathrm{e}}$ siècle, des corps nationaux d'ingénieurs a facilité l'incorporation de certains ingénieurs, mais a contraint les "laissés pour compte » à rechercher un emploi dans un autre pays. La rédaction d'un traité qui permet la démonstration de savoirs théoriques et pratiques, constitue parfois un outil essentiel pour la recherche d'un nouvel employeur.

Cette présentation ne se veut pas exhaustive mais souhaite, à partir d'exemples, proposer un état de la question en mettant en évidence, d'une part, des stratégies d'auteurs, d'autre part, des politiques de traduction.

\section{Les traductions, reflets de la mobilité des ingénieurs militaires et des stratégies d'auteurs}

Les ingénieurs écrivent souvent pour faire connaître leurs compétences, particulièrement auprès des princes, rois ou hauts commanditaires qui les emploient ou sont susceptibles de le faire. Ils peuvent modifier la langue de publication selon les circonstances, pour la recherche d'un mécène, ou en fonction de la finalité de l'ouvrage, qu'il s'agisse de la divulgation de nouveautés ou d'une synthèse destinée à la formation des ingénieurs.

Les ingénieurs italiens $\mathrm{du} \mathrm{xvI}^{\mathrm{e}}$ siècle, très recherchés dans toute l'Europe, ont publié en italien, ce qui a limité la diffusion de leurs ouvrages. Leurs traités sont cependant présents dans les bibliothèques des nobles ou des ingénieurs européens, comme en témoignent les exlibris des ouvrages conservés à la bibliothèque Mazarine, à l'exemple de ceux du médecin Girolamo Maggi et du capitaine Jacomo Castriotto, Della fortificatione delle citta, Venise, $1564^{4}$.

La cour de France, qualifiée "d'italienne » dans l'entourage de Catherine de Médicis, constitue en cela une clef de compréhension de la fréquence de la pos- session d'ouvrages en italien dans les bibliothèques de nobles français (notamment à partir de l'avènement d'Henri II, son époux, en 1547 jusqu'à la fin de sa régence officielle puis officieuse en 1574, avec le retour de Pologne de son fils Henri III). Des livres peuvent circuler sans être traduits, associés à d'autres textes dans des recueils collationnés. Citons un recueil du $\mathrm{xvII}^{\mathrm{e}}$ siècle, conservé à la bibliothèque Mazarine, comprenant la Difesa o vero Riposta de Silvio Maggieri (1639), dédicacée au prince D. Tadeo Barberini, préfet de Rome et généralissime de la sainte Église, le Traité d'escrime de Hyeronimo Cavalcabo, traduit en français par Villamont (Paris, 1595), et une Instruction sur le fait d'artillerie, circonstances et dépendances d'icelle et sur quoy les officiers sont ordinairement interrogés par un grand maitre ${ }^{5}$. Les ouvrages d'ingénieurs italiens réputés sont néanmoins traduits, comme El Capitan de Girolamo Cataneo (Brescia, 1564), traduit en français à Lyon en 1574, 1589 et 1593, puis en latin et en français en 1600, avant une traduction allemande en $1606^{6}$. D'autres traités italiens traduits en français pourraient être cités, comme 
ceux de Girolamo Zanchi (1516-1590) ou de Carlo Theti (1529-1589).

Les traductions peuvent être facilitées lorsque ces ingénieurs servent pendant plusieurs années dans un pays. Ainsi, Aurelio di Pasino de Ferrare (c. 15101584), au service du duc de Bouillon, publie à Anvers en français un Discours sur plusieurs poincts de l'architecture de guerre, concernant les fortifications tant anciennes que modernes (1578), ou Augustino Ramelli (1531, Milan-1600), après une carrière italienne, passe au service du roi Henri III et publie à Paris, en 1588, en français et en italien, Diverses et artificieuses machines, traduites en allemand, à Leipzig, en 1602. Le Romain Pietro Sardi qui travaille dès 1604 en Espagne, participe à diverses campagnes et publie six traités d'architecture militaire et d'artillerie entre 1618 et 1642 ; son ouvrage Corno dogale della architettura militare, 1618, est traduit par J. de Zetter, en français et en allemand, à Francfort, en $1623^{7}$.

$\mathrm{Au}$ début du $\mathrm{XvII}^{\mathrm{e}}$ siècle, les ingénieurs de langue française se font plus nombreux et leur mobilité est attestée. Le célèbre Dieppois Salomon de Caus (1576-1626) exerce ses talents d'architecte et d'ingénieur, en particulier dans le domaine de l'hydraulique, au service de plusieurs cours européennes. Précepteur du prince Henry de Galles, il publie en français, à Londres, La perspective avec la raison des ombres et miroirs (1611); l'ouvrage est aussi édité à Francfort, toujours en français. Cependant, lorsque Salomon passe au service de l'Électeur Palatin Frédéric $\mathrm{V}$, avec la fonction d'ingénieur des bâtiments et jardins, l'ouvrage qu'il publie à Francfort, chez Jan Norton en 1615, Les raisons de forces mouvantes avec diverses machines tant utilles que plaisantes - un des premiers traités de mécanique appliquée essentiellement à l'hydraulique - est immédiatement traduit en allemand, chez le libraire français Abraham Pacquart, avec une dédicace à la princesse Élisabeth, fille de Jacques $\mathrm{I}^{\mathrm{er}}$ Stuart et épouse de Frédéric $V^{8}$. Cette traduction simultanée s'explique par le lieu de publication (Francfort) et la langue de l'auteur.

Un autre Français témoigne de l'usage du latin, en fonction du sujet abordé, du dédicataire et $\mathrm{du}$ lieu de publication. L'ingénieur toulousain Antoine de Ville (1596-1657) a servi successivement le roi de France et le duc de Savoie qu'il a suivi aux Pays-Bas en 1626. Il a voyagé en Italie centrale et dans le Royaume de Naples, avant de passer, en 1632, au service de la République de Venise, pour laquelle il a effectué de nombreuses missions d'inspection aux confins de la République. C'est en Italie qu'il compose son premier grand ouvrage, Les fortifications, écrit en français et publié chez Irénée Barlet, à Lyon, en 1628, réédité cinq fois au cours du $\mathrm{XVII}^{\mathrm{e}}$ siècle, à Paris, Lyon et Amsterdam? Au cours du Xvir e siècle, des passages de ce traité sont traduits dans des ouvrages allemands et anglais sur les fortifications. La première traduction intégrale ne paraitra qu'en 1760 à Francfort ${ }^{10}$. De Ville est un homme cultivé qui écrit en latin plusieurs petits ouvrages ${ }^{11}$ avant d'offrir à Louis XIII, après son retour en France, la relation du siège de Corbie, en latin ${ }^{12}$, hommage au souverain d'un homme de guerre et de lettres.

Ses publications suivantes sont celles d'un ingénieur, maître dans l'art des sièges. Elles sont rédigées en français pour une large diffusion : Le siège de 
Hesdin (Lyon, 1639) et De la charge de gouverneurs des places (Paris, 1639) avec une dédicace au cardinal de Richelieu. Ce dernier ouvrage, bien que consacré à la logistique militaire, est accompagné d'un abrégé de la fortification. Il sera réédité en 1640, 1656 et 1666, puis revu et corrigé à Amsterdam, chez Abraham Wolfgang, en 1674, et enfin traduit en néerlandais à Amsterdam, en 1676. La réputation d'Antoine de Ville est encore vive en 1685 , notamment chez ses homologues d'autres pays européens, comme Johann Jacob Werdmüller qui traduit l'ouvrage en allemand $^{13}$. Ce dernier est ingénieur, auteur du Der Ingenieure Probierstein (Georg Schiede, 1685) et déjà traducteur du traité de fortifications de l'ingénieur français Blaise Pagan, en $1684^{14}$. De même, la traduction portugaise du Gouverneur des places est due à l'officier ingénieur Antonio Pedrozo Galram, en 1708, en pleine guerre de succession d'Espagne ${ }^{15}$. La traduction est assurée par un ingénieur qui maîtrise les termes techniques et est capable de comprendre les démonstrations et les nouveautés d'un ouvrage de fortifications, compétence qui est susceptible d'assurer un plus grand succès à la traduction.

La mobilité des ingénieurs est une clef de compréhension des lieux d'édition de leurs ouvrages et des traductions quasi simultanées dans la langue du pays dans lequel ils sont employés. Certains sont toutefois contraints de publier en plusieurs langues, tels les Espagnols qui servent dans les Pays-Bas espagnols. Ainsi, le grand ingénieur espagnol, directeur de l'Académie royale et militaire des Pays-Bas, Sebastian Fernandez de Medrano, a publié à Bruxelles El Ingeniero (L. Marchant, 1687). Quelques années plus tard en 1696, il donne des précisions intéressantes dans la préface de l'édition française, L'ingénieur pratique ou l'architecture militaire et moderne, contenant la fortification régulière et irrégulière [...] la manière d'attaquer et défendre une place [...] qu'il dédie à Joseph Ferdinand, prince électoral de Bavière :

«Quantité de personnes tant de ses sujets que d'autres qui ignorent la langue espagnolle, ayant trouvé beaucoup de difficulté à bien comprendre tout le fort des différents livres que j'ay mis au jour... je n'ay pû refuser aux pressentes solicitations qu'ils m'ont faites, pourque $\mathrm{du}$ moins je voulusse permettre $q^{\prime}$ 'on traduise en langue Françoise, le Traité de ma Fortification et de ma Geometrie; mais après avoir commis ce soin à deux ou trois personnes intelligentes dans l'une ou l'autre langue, j'ai trouvé que dans ce qu'ils avaient commencé à traduire, la doctrine et les maximes contenues dans l'original espagnol n'y etoient pas expliquées assez clairement etc c'est ce qui a été cause que je n'ai pas voulu que l'on poursuivit la traduction; mais mes disciples s'étant opiniatrés de nouveau à m'en solliciter, j'ai à la fin résolu de la faire moy-même et d'y ajouter quantité de choses dont il n'est pas parlé dans l'espagnol. Je me suis dépouillé de tout amour propre, et j'ai entrepris cet ouvrage, moy qui suis espagnol de naissance et peu versé dans la manière d'écrire des François ${ }^{16}$.» 
Publier dans la langue de son protecteur et patron est aussi une stratégie fréquente de la part d'ingénieurs qui savent que leur emploi dépend du bon vouloir du prince. C'est pourquoi il est intéressant de suivre les différentes éditions d'un même ouvrage et les épîtres dédicaces qui les introduisent.

Les libraires des Provinces-Unies sont toujours à la recherche de manuscrits traitant de sujets demandés par un lectorat éclairé et intéressé par les écrits des ingénieurs. Les ouvrages en bas allemand ou en hollandais sont souvent rapidement traduits en français, en raison de l'intérêt pour ces techniques en France, mais aussi parce que les lecteurs peuvent être plus nombreux dans le pays le plus peuplé d'Europe. La présence de huguenots réfugiés dans les villes de Leyde, Amsterdam ou La Haye permet une traduction rapide en français ou $a$ contrario en néerlandais ${ }^{17}$.

L'un des traités de fortifications les plus répandus dans l'Europe du début du XVII ${ }^{\mathrm{e}}$ siècle est la Fortification ou architecture militaire tant offensive que defensive, de Samuel Marolois, mathématicien et ingénieur (c. 1572-1627), descendant de huguenots français établis en Hollande. L'ouvrage est publié, en 1614, à La Haye, chez H. Hondius, comme une partie de Opera mathematica ou OEuvres de mathématiques traictans de Geometrie, perspective, architecture et fortification par l'éditeur amstellodamois Jan Janssen. Il a été corrigé et augmenté en français par Albert Girard, mathématicien et ingénieur des armées de Frédéric de Nassau. Albert Girard en a assuré les traductions en néerlandais en 1627-1628, puis en latin en $1638^{18}$.
Il est possible de mettre en évidence les liens entre la publication et la traduction d'un ouvrage et les stratégies de carrière d'un ingénieur, en s'intéressant, par exemple, à Mathias Dögen, dont le cas n'est pas singulier et reflète la nécessité pour un ingénieur d'intégrer la clientèle d'un puissant commanditaire. L'ingénieur Matthias Dögen (1605/16061672) est originaire de Drambourg, en Haute-Saxe. Peu d'informations sur sa famille et sur sa formation subsistent, mais on sait qu'il a suivi les cours de la fameuse école de mathématique de Leyde, la Duytsche Mathematique, fondée en 1600 par Maurice de Nassau pour la formation des mathématiciens, des ingénieurs militaires et des arpenteurs-géographes, et dontles cours ontété organisés par Simon Stevin ${ }^{19}$. Il entre rapidement au service des Provinces-Unies comme quartier-maître dans l'amirauté, ce qui le rapproche du Stathouder Frédéric-Henri de Nassau, prince d'Orange, qui l'encourage à publier son ouvrage Architectura militaris moderna en $1647^{20}$.

Cette première édition en latin est réalisée chez le célèbre imprimeur d'Amsterdam, Louis Elzevier, et l'épître dédicatoire est adressée au prince. La mort de ce dernier, le 14 mars 1647, contraint Mathias Dögen à se placer sous la protection de son fils et successeur, Guillaume II d'Orange, auquel il dédie l'édition française de 1648, assurée aussi par L. Elzevier. Toutefois, Matthias Dögen est resté fidèle à son " souverain naturel ", l'électeur de Brandebourg Georges Guillaume I Hohenzollern, qui l'emploie comme agent diplomatique et auquel il adresse chaque semaine des informations sur les événements survenus à Amsterdam. En 1640, il renou- 
velle sa fidélité à son fils et successeur, Frédéric-Guillaume, qui renforce ses liens avec les Provinces-Unies en épousant, en 1646, Louise d'Orange-Nassau, fille de Frédéric-Henri de Nassau. À l'occasion de la venue, pour une mission diplomatique, de l'électeur à La Haye en 1648, Matthias Dögen lui remet et lui dédicace la partie de son ouvrage en latin consacrée à l'attaque et à la défense des places, puis la traduction allemande, sa langue maternelle, éditée par Elzevier la même année.

L'important rayonnement de l'ouvrage de Matthias Dögen tout au long de la seconde moitié du $\mathrm{xvII}^{\mathrm{e}}$ siècle s'explique en grande partie par la politique de traduction suivie par l'éditeur Louis Elzevier qui a demandé, en 1648, une traduction à l'écrivain huguenot vivant en Hollande, Helie Poirier, connu pour ses poèmes. L'ouvrage circulant en français et en allemand était assuré d'un large lectorat et l'auteur, bien qu'alle- mand, faisait honneur à la formation dispensée par l'école mathématique de Leyde $^{21}$. La fidélité de Dögen à FrédéricGuillaume, surnommé le Grand, est récompensée. L'électeur, décidé à entreprendre de grands travaux, fait appel à l'ingénieur qui revient ainsi en Brandebourg au début des années 1650, avec le titre de conseiller grand-ducal pour les fortifications. Il participe en 1658 et 1659 , aux travaux de fortification de Berlin sous la direction de l'architecte autrichien Johan Gregor Memhardt et à la création de la flotte de Brandebourg décidée par le prince électeur.

Les exemples de Marolois et de Dögen attestent du maintien du latin dans certaines éditions d'ouvrages d'ingénieurs de la première moitié $d u x{ }^{2}{ }^{e}$ siècle, tandis que les éditions en flamand (ou bas allemand) et français tendent à devenir progressivement la règle au milieu du siècle.

\section{Les politiques de traduction et leurs limites}

Les stratégies personnelles d'édition et de traduction ont été possibles grâce à certains éditeurs qui en ont assuré les publications et ont compris l'intérêt des éditions simultanées en plusieurs langues.

La politique de publication des Elzevier, illustre famille de typographes néerlandais d'origine brabançonne (de Louvain), très actifs au $\mathrm{xVII}^{\mathrm{e}}$ siècle à Leyde (ils sont imprimeurs-jurés de l'université de Leyde) et à Amsterdam, servira d'exemple pour mettre en évidence quelques traits caractéristiques des politiques de traduction des ouvrages d'ingénieurs.

Les Provinces-Unies, carrefour de l'Europe occidentale, premier pays à développer une formation pour les ingénieurs et terre d'accueil pour les huguenots français, sont favorables aux ingénieurs ambitieux. Une famille d'imprimeurs-libraires émerge au XVII ${ }^{\mathrm{e}}$ siècle, les Elzevier. Spécialisés dans les éditions des classiques latins dans le format in-12, ils impriment aussi des ouvrages en français, soit des copies d'ouvrages régulièrement publiés en France avec privilège, 
soit des éditions originales de textes considérés comme subversifs ou licencieux (les petits in-12 peuvent aisément circuler en contrebande, en feuilles, dans des tonneaux, par exemple), ou dont les auteurs souhaitent tester l'accueil de leurs écrits par le public avant de les faire éditer en France. Proches de l'école mathématique de Leyde, ils y éditent en français les ouvrages scientifiques de Simon Stevin ou encore de Galilée, par exemple les Discours concernant deux sciences nouvelles, en $1638^{22}$.

Bonaventura Elzevier et Abraham Elzevier publient plusieurs écrits d'ingénieurs à l'image de ceux déjà mentionnés d'Antoine de Ville en 1640, le traité en plusieurs langues de Matthias Dögen en 1648, mais aussi, déjà en 1643, l'ouvrage Elementorum architecturae militaris Libri I, dédié à Frédéric III, futur roi de Danemark et de Norvège, du silésien Nicolaus Goldman (1611-1665), professeur à l'école de Leyde. Deux ans plus tard, Elzevier édite la traduction française, La nouvelle fortification, que l'auteur dédicace au Stadhouder Frédéric-Henri de Nassau ${ }^{23}$.

Le latin est progressivement abandonné dans les ouvrages techniques dès le début du XviI ${ }^{\mathrm{e}}$ siècle, notamment dans les Provinces-Unies, les Pays-Bas espagnols et la France, alors qu'il se maintient plus nettement pour les ouvrages scientifiques, notamment en Angleterre et dans l'Empire $^{24}$. Les Elzevier s'adaptent et assurent dans la seconde moitié du siècle les publications et traductions du hollandais, de l'allemand et du français, mais ils ne dominent plus le marché du livre technique.

La notoriété des ingénieurs français assure le succès de leurs publications dans une Europe qui craint et admire le royaume de France. Contrepartie parfois regrettable, les lecteurs qui veulent percer les secrets et les faiblesses éventuelles de ces ingénieurs sont les clients assurés d'éditeurs parfois peu scrupuleux sur l'exactitude du texte ou son attribution.

Témoigne de cette notoriété François Blondel, architecte puis ingénieur du roi, nommé, en 1656, lecteur de mathématiques et de fortification au Collège Royal et, en 1673, précepteur du Grand Dauphin pour les mathématiques. Il a fait circuler une forme manuscrite de sa théorie sur les fortifications, publiée sous le titre de Nouvelle manière de fortifier les places, à Paris, chez Nicolas Langlois, en 1683. La publication est rapidement suivie d'autres éditions en français à La Haye (1684, 1686, 1688, 1711), Londres (1684), Paris $(1693,1699)$ et Amsterdam $(1699)^{25}$. Deux traductions seulement ont été effectuées alors que le livre est présent dans de nombreuses bibliothèques d'Europe en français : une traduction allemande, à Nuremberg, chez Johann Hoffmann, en 1686, puis une traduction russe par Ivan Zotov, à Moscou, en $1711^{26}$. Il est remarquable que le livre circule essentiellement en français, signe possible de la domination des théoriciens français de la fortification.

Le deuxième auteur très prisé est Alain Manesson-Mallet, ingénieur français qui a servi au Portugal avant d'être nommé maître de mathématiques des pages de la petite Écurie du roi de France. Une première publication vraisemblablement pirate d'un traité, Les travaux de Mars, ou la fortification nouvelle, est faite à Paris, Jean Hénault \& Claude Garbin, 1671-1672, et à Amsterdam, chez Jacob 
von Meurs qui publie la même année une traduction allemande et une autre hollandaise ${ }^{27}$. Devant le succès, l'auteur revoit son texte et le publie simultanément sous le titre des Travaux de Mars ou l'art de la guerre [...] à Paris (D. Thierry, 1684-1685) et Amsterdam (Jan et Gillis Janson, à Waesbergue \& Compagnie), dans une impression moins soignée mais qui a servi à la traduction russe par Zotov sur les presses du tsar Pierre Le Grand en $1720^{28}$. L'ouvrage a été deux autres fois traduit en hollandais (Amsterdam, Wolfgang Waasbergeb, Boom, von Someren und Goethals, 1687, et Leyde, Pieter van der Aa, 1695) et réédité en français à Amsterdam, Henri Desbordes, 1696, et à La Haye, Adrin Moetjens, 1696.

Cependant, la notoriété d'un ingénieur peut conduire les libraires-imprimeurs qui sont à la recherche de ventes lucratives, à publier des textes faussement attribués ou approximatifs. Un exemple permettra de saisir les difficultés d'attribution des textes et le succès d'auteurs qui se font une spécialité de faire connaître la méthode du célèbre ingénieur français, Vauban.

En l'absence de publication de Vauban sur la fortification, ce sont d'autres auteurs qui ont repris à leur compte son savoir-faire en architecture militaire. En Angleterre, la connaissance dans ce domaine et dans celui de la guerre de siège est considérée par l'aristocratie anglaise de la fin du $\mathrm{xvII}^{\mathrm{e}}$ siècle comme nécessaire à la formation de sa jeunesse. Les ouvrages anglais portant sur le savoir des ingénieurs militaires consacrent à Vauban un chapitre ${ }^{29}$ et les auteurs qui sont traduits en anglais se présentent toujours comme ses élèves ou comme connaisseurs de sa méthode de fortifica- tion. C'est le cas de l'abbé Du Fay, Manière de fortifier selon la méthode de Mr de Vauban, Paris, Coignard, 1681, ou du chevalier de Cambray, Manière de fortifier de M. de Vauban, Amsterdam, Pierre Mortier, 1689, réédités sous leurs deux noms à Paris (Vve Cramoisy, 1694) ${ }^{30}$. L'ouvrage du chevalier de Cambray est traduit par Abel Swall, à Londres, dès 1691, avec des éditions progressivement enrichies en $1693,1702,1748$ et $1762^{31}$, puis c'est le tour de La manière de fortifier d'après Vauban de l'abbé du Fay, associée à un traité de fortification de Jacques Ozanam revu par Jacques Desaguliers, en $1711^{32}$. Toujours de seconde main, les écrits d'un autre huguenot exilé, Abel Boyer, accordent une place prépondérante à Vauban. Il écrit, en 1701, The Draughts of the Most Remarkable Fortified Towns of Europe, avec une dédicace à Henry, Earl of Galloway, "protecteur des refugiés religieux français ${ }^{33} »$. Ainsi, Vauban est connu en Angleterre par la traduction ou l'édition de ces traités de deuxième main $^{34}$, comme il l'est en Allemagne, en Espagne et en Italie ${ }^{35}$.

Quand l'enseignement de la fortification est régulièrement assuré à l'Académie royale de Woolwich créée en 1741, le texte de Charles Goulon (ou le Goulon ou Goullon), un des élèves de Vauban, comprenant Mémoires pour la défense et l'attaque des places, augmenté de deux mémoires de Vauban, " La relation du siège d'Ath » et le " Directeur des fortifications ${ }^{36}$ ", édition de 1730, est traduit à Londres en 1745. Charles Goulon, huguenot originaire de Metz, capitaine des mineurs, a quitté la France en novembre 1685 et est passé au service de Guillaume d'Orange, puis à celui de l'empereur Habsbourg. Lorsque 
son texte est publié, en 1706, à Wesel, dans la Nouvelle fortification, tant pour un terrain bas et humide, du baron van Coehoorn, il possède le titre d'ingénieur et généralissime de l'empereur. Outre de nombreuses éditions à Amsterdam et à La Haye, les traductions allemandes se sont succédées et une traduction en suédois de 1728 atteste du crédit accordé à l'auteur dans la détention des savoirfaire de Vauban ${ }^{37}$.

Il est instructif de constater que le traité Architectura civili-militaris (Nuremberg, 1702) du grand ingénieur allemand L.C. Sturm, qui a eu de très nombreuses rééditions allemandes jusqu'en 1755, puis deux traductions suédoises en 1794 et 1795, n'a pas été traduit en français. En revanche, son Der wahre Vauban oder der von den Deutschen und Holländer verbesserte franzoösische Ingenieur (1703) a été traduit à La Haye (N. Wilt, 1708 et 1710) sous le titre Le véritable Vauban se montrant au lieu du faux Vauban. La mention du nom de Vauban dans le titre impose la nécessité d'une traduction, même si Sturm y développe ses propres idées. Les ouvrages de fortification allemands n'étant pas traduits en français, Sturm utilise cette forme de supercherie.

Comment comprendre la publication à La Haye, en 1685, chez Van Bulderen, du Directeur des fortifications de Vauban? Ce dernier n'a jamais voulu publier l'ouvrage, mais le manuscrit a probablement été remis au libraire par un ingénieur huguenot, l'année de la révocation de l'édit de Nantes. C'est à La Haye aussi qu'en 1737, trente ans après le décès de Vauban, sera publié, avec beaucoup d'erreurs, un Traité de l'attaque et de la défense des places par le maréchal de Vauban. Le libraire hollandais Pierre de Hondt qui avait obtenu une (mauvaise) copie du manuscrit du "Traité de l'attaque des places » rédigé en 1704 pour le duc de Bourgogne, petit-fils de Louis XIV, a publié, à la suite, un mémoire sur la Défense des places qu'il attribue sans vérification à Vauban. On sait que le maréchal a composé, en 1706, de sa propre initiative, un mémoire sur la défense des places resté inachevé en raison de son décès. La « Défense des places » n'a pas circulé sous forme de copies, aussi le libraire a-t-il pris (en toute bonne foi?) le manuscrit portant le même titre et rédigé par l'ingénieur Guillaume de Lafon de Boisguérin, seigneur des Houlières (1621-1694), pour celui de Vauban. Ce dernier connaissait l'écrit de l'ingénieur qui avait travaillé sous sa direction à la citadelle de Lille et à celle de Tournai ${ }^{38}$, mais il en récusait le contenu, comme l'atteste le jugement sans appel en marge du manuscrit conservé dans ses archives : "Cet écrit est de quelqu'un qui a de l'esprit et ne s'explique pas mal et qui a vu quelques sièges, soit pour attaquer ou pour défendre, mais qui n'a nul principe, et qui, en un mot, n'est pas ingénieur ${ }^{39}$. »

L'erreur d'attribution du texte se poursuit dans l'édition suivante par Jean et Herman Verbeeck, à Leyde, en $1740^{40}$. Le nom de Vauban assurant un succès de vente pour les libraires, ceux-ci publient sans vérification des écrits qui lui sont attribués ${ }^{41}$. Le Traité de la Défense des places qui n'est pas de Vauban, a été traduit en une quinzaine de langues dont l'espagnol, en 1743, par l'ingénieur Ignacio Sala qui a fait confiance aux libraires de La Haye et a commenté de manière critique le mémoire, y ajoutant même des nouveautés aux paragraphes 4,5 et $6^{42}$. 
Il faudra attendre l'édition critique du baron Valazé, en 1829, pour que le véritable Traité de défense des places de Vauban soit connu ${ }^{43}$.

La rivalité entre les ingénieurs emblématiques de techniques " nationales » à la fin $d u x \mathrm{xII}^{\mathrm{e}}$ siècle encourage les libraires sans scrupules à faire circuler des ouvrages erronés. L'ingénieur hollandais Menno Van Coehorn en est un exemple. Un article récent de Philippe Bragard permet de mieux connaître celui qu'on a surnommé le Vauban hollandais ${ }^{44}$. Il a dirigé les sièges pour Guillaume d'Orange, Stathouder des Provinces-Unies puis roi d'Angleterre, lors de la guerre de la Ligue d'Augsbourg puis de Succession d'Espagne, et a publié Nieuwe vestingbouw op een natte of lage horisont [...] à Louvain, en $1685^{45}$. La traduction française, Nouvelle fortification, tant pour un terrain bas et humide que sec et élevé, La Haye, 1706, est dénoncée par l'abbé Deidier, en 1742, comme mauvaise, tout comme le texte original « écrit dans un néerlandais parfois obscur »:

" À peine s'est-on engagé dans cette lecture qu' on y trouve tant de manières obscures de parler, qui viennent peut-être de la part du traducteur peu versé dans la langue, si peu d'éclaircissemens touchant les constructions que l'auteur ne se donne pas la peine de détailler, tant d'affectation de cacher sa manière de faire les revêtemens, et les chicanes qu'il pourroit ajouter dans ses ouvrages, un détail si ennuyeux de la défense qu'on peut opposer, selon les différentes attaques qu'on y feroit à ses places, et enfin des parallèles si longs de ses systèmes avec ceux de M. de Vauban, qu'on n'a ni le courage ni la patience de parcourir cet ouvrage jusqu'au bout ${ }^{46}$.»

Philippe Bragard ajoute ${ }^{47}$ : « Et force est de constater que même le titre français trahit l'original dans lequel il n'est pas question de terrain "sec et élevé". " Malgré ces difficultés de compréhension, l'ouvrage est publié par J.V. Wesel en français, en 1705, puis en allemand, en 1708 (et 1718), de nouveau en allemand à Düsseldorf, en 1709, mais aussi en anglais, à Londres, en 1705 et 1725, et enfin en russe, à Moscou, en 1709.

Nous avons vu que les imprimeurslibraires hollandais ont souvent assuré les traductions en français des ouvrages hollandais et même allemands grâce aux huguenots, alors que les imprimeurs-libraires français n'avaient pas publié d'ouvrages étrangers, certainement à cause du plus grand nombre et de la réputation des ingénieurs français. Ainsi, les ouvrages allemands les plus connus, comme ceux de Georg Rimpler qui publie, dès 1671, Kriegs-Bau-Kunst, à Frankfurt, réédité en 1674, 1678, 1719, ceux de L. N Sturm vus précédemment ou ceux des ingénieurs anglais, de Paul Ive (1689) à John Muller (1746, avec huit éditions de 1755 à 1807) ne sont pas traduits en français, alors que ce dernier, par exemple, est traduit en espagnol par D.M. Sanchez Taramas (T. Piferrer, $1769)^{48}$.

Cependant, la politique d'édition et de traduction de certains souverains peut attester de la pénurie d'ingénieurs dans leur royaume et de l'aire d'influence des autres pays. C'est le cas de la Russie du tsar Pierre ${ }^{\text {er49 }}$. Si nous prenons l'exemple 
de la fortification, cinq auteurs sont traduits entre 1708 et 1711, reflet de l'influence des Hollandais et des Allemands sur le tsar ${ }^{50}$, alors que la méthode de Vauban d'après l'abbé du Fay ne sera traduite qu'en 1724, à Saint-Pétersbourg, par Vasilij Suvorov (1705-1775), ordonnance et traducteur du tsar, sous un titre que l'on peut traduire par Bonne manière de fortifier les villes ${ }^{51}$.

\section{Conclusion}

Les traductions des ouvrages de certains ingénieurs militaires reflètent la réputation de ces derniers comme poliorcètes, théoriciens de la guerre, architectes ou techniciens hydrauliques. Le latin qui a été la langue commune des ingénieurs $\mathrm{du} \mathrm{xvI}^{\mathrm{e}}$ siècle, se maintient jusqu'à la première moitié $d u X{ }^{e} I^{e}$ siècle dans l'aire germanique, mais le français est très souvent la langue de traduction de ces ouvrages. Le pays le plus peuplé d'Europe acquiert, sous Louis XIV, une suprématie dans l'art des sièges et de la fortification au cours d'un long règne marqué par l'omniprésence de la guerre et par un programme de construction de places fortes inégalé. Plusieurs centaines d'ingénieurs ont été recrutés et ont servi en France, faisant de ce royaume un pôle attractif pour ceux qui souhaitent publier. Les éditeurs savent qu'il existe un lectorat francophone conséquent, puisque le français devient la langue des élites, d'où leur stratégie de traduction. A contrario, les éditeurs de traités français n'ont pas jugé nécessaire de les traduire en d'autres langues.
Les traductions ont été difficiles en raison des lacunes en vocabulaire technique de la langue russe. Les premières traductions comportent beaucoup de termes allemands, impossibles à traduire, et par la suite les termes de la fortification, de l'artillerie et de l'hydraulique sont empruntés au français, autre signe de la prééminence des ingénieurs français.

\section{Notes}

1. C'est ce qui apparaît à Paris pour les livres scientifiques, voir notamment Sabine JuRATIC, "Publier les sciences au $18^{\mathrm{e}}$ siècle : la librairie parisienne et la diffusion des savoirs scientifiques ", Revue du xVIII siècle, La République des sciences. Réseaux des correspondances, des académies et des livres scientifiques, $\mathrm{n}^{\circ} 40,2008$, p. 301-313. Les Elzevier à Leyde et Amsterdam, les Jombert à Paris ou Z. de Zetter à Francfort sont des exemples de ces libraires.

2. Les études qui traitent de la traduction à la période moderne ne se sont pas intéressées à ces livres techniques avant la publication dirigée par Yves Chevrel, Annie CoIntre et Yen-Maï Tran-Gervat, Histoire des traductions en langue française, XVII et XVIII siècles (1610-1815), Lagrasse, Éditions Verdier, 2014; voir Patrice BRET et Ellen Moerman, «Sciences et arts ", notamment p. 595722.

3. Sur l'interaction entre auteurs et éditeurs, Roger CHARTIER, La main de l'auteur et l'esprit de l'imprimeur, XVI ${ }^{e}-X V I I l^{e}$ siècle, Paris, Gallimard, Collection Folio histoire ( $\left.n^{\circ} 243\right), 2015$.

4. L'exemplaire de la bibliothèque Mazarine, $2^{\circ} 4816$ D, est au chiffre du cardinal de Richelieu, l'exemplaire de la Bnf est à celui de Gaston d'Orléans. Notons que le Raggiomento sopra le fortezze della Francia (Venise, 1588), de Girolamo Castriotto, a été traduit en allemand : Raisonnement über die französischen Festungen, Giessen, 1620.

5. Difesa, o vero Riposta ad alcune obbiettioni fatte alla forticatione italiana da diversi, de Silvio Maggieri d'Urbino, all' illustriss. Ed eccellentiss. Signor, e padron moi colendissimo il sig. Principe D. Tadeo Barberini prefetto di Roma, e Generalissimo di S. Chiesa, Roma, per 
Antonio Landini, 1639,111 p. in- $12^{\circ}$, sans dessins ni plans; le traité d'escrime de Hyeronime Caluacabo, de Bologne, est traduit par le seigneur de Villamont, chevalier de l'ordre de Jérusalem, à Paris, chez Claude Montr'œil et Jean Richer, 1595. Dédicace au comte de Brisac, maréchal de France, 27 p. ; 1'Instruction sur le fait d'artillerie, circonstances et dépendances d'icelle et sur quoy les officiers sont ordinairement interrogés par un grand maître, avec privilège, Paris, 1636.

6. Le capitaine de Jerosme Cataneo contenant la manière de fortifier les places assailir et défendre, tout revue, corrigée et augmentée par l'auteur, Lyon, de Tournes, 1574; Lyon, 1589; Lyon, Jacques Roussin, 1593. À Lyon, Jean de Tornes publie, en 1600, en latin Hier. Cataneus de arte bellica, et en français. En allemand, Herrn Cataneos Neu Gespräch..., Eisenach, 1606.

7. Couronne impériale de l'architecture militaire, c'est-à-dire Vraye et artificieuse introduction à la fortification pour tous lieux et contre tous les efforts de l'assaillant, par Pierre Sardi, [...] traduit en langue françoise. Sur la fin sont adjouttez quelques principes de la géométrie pris des escripts d'Euclyde servans à l'architecture militaire, Francfort, impr. par H. Paltheni, 1623, aux frais de J. de Zetter, éditeur et traducteur., in-fol., pièces limin., 83 p., pl., BnF V 2283.

8. Les raisons des forces mouvantes auec diverses machines tant utilles que plaisantes ausquelles sont adjoints plusieurs desseings de grotes et fontaines, par Salomon de Caus, ingenieur et architecte de son altesse Palatine electorale, par W. Richter, à Francfort en la boutique de Jan Norton, 1615, traduit en allemand Von gewaltsamen Bewegungen : Beschreibung etlicher, so wol nützlichen alß lustigen Machiner [...] Durch Salomon de Caus..., Francfort, Abraham Pacquart, 1615.

9. On trouve l'ouvrage dans la plupart des grandes bibliothèques de l'époque et il est également exporté dans les colonies. Il demeurera un grand classique du genre jusqu'au xvIII $^{\mathrm{e}}$ siècle; Leblond l'étudie longuement dans l'article "Fortification » de l'Encyclopédie.

10. Klaus JoRdAN, Bibliographie zur Geschichte des Festungsbaues von den Anfängen bis 1914, Marburg, 2003, p. 281.

11. Antonii Deville [...] Portus et urbis Polae antiquitatum, et Thynnorum piscationis descriptio curiosa, figuris aeneis [...] illustrata, Venetiis, Pinelliana, 1633. Ces deux textes ont été réédités en 1722 dans un recueil : Thesaurus antiquitatum et historiarum Italiae, Lugduni Batavum, P. Vander Aa., t. 6; en 1634, il publie, à Venise, Pyctomachia veneta seu Pugnorum certamen Venetum, exposé savant sur les origines et les traditions de ces pugilats et combats organisés au cours de fêtes à Venise.
12. Obsidio Corbiensis dicata Regi. Ab Antonio de Ville [...] cum figuris a Michaële van Lochom, Parisiis, apud viduam N. Buon, via Jacobea sub signo sancti Claudii juxta Mathurinenses, M.DC.XXXVII [1637].

13. Des Commandantenspiegels, oder gründlicher Unterrichtung, wie ein Commandant seinen Platz wider alle feindliche Anschläg und Surprises, wie auch wider die [...] Belägerungen defendiren solle, anderer Theil, Johann Jacob Werdmüller, Éditeur Schiele ou Schiede, 1685.

14. Blaise-François de PAGAN, DeßGrafen von Pagan Neuer Vestungs-Bau. Übersetzt von Werdmüller, Frankfurt a. M. u. Leipzig, J.G. Drullmann, 1684. Quelques années plus tard, il publie aussi une synthèse de l'art de la fortification : Johann J. Werdmüller, Apologia Fortificatoria oder Schutzrede [...] Schauplatz der alten und neuen Fortifications maximes, Frankfurt/Main, Görlien, 1691.

15. O governador de praça, éd. Na officina de Antonio Pedrozo Galram Galrão, 1708.

16. Préface de l'édition française, L'ingénieur pratique ou l'architecture militaire et moderne, contenant la fortification régulière et irrégulière... la manière d'attaquer et défendre une place..., Bruxelles, L. Marchant, 1696. Les plans dessinés par l'ingénieur flamand Verboom dans l'édition espagnole sont repris dans l'édition française.

17. Notons qu'avant même la création de l'école de Leyde, un opuscule de Stevin, De Havenvinding, écrit en bas allemand, avait été traduit en français, à Leyde, en l'imprimerie de Plantin, par C. de Ravelengien, 1599 , in- $4^{\circ}, 30$ p., sous le titre $L e$ Trouve-port.

18. S. MAROLOIS, Fortification vermehret, gebessert und erlaütert durch, Albert Gerhart [sic], Amsterdam, J. Janssen, 1627. Les éditions française de 1628 et latine de 1638 sont faites chez le même éditeur.

19. Sur cet établissement créé par Maurice de Nassau et Simon Stevin, C. VAN DEN Heuvel, Le traité incomplet de l'art militaire et l'instruction pour une école des ingénieurs de Simon Stevin, dans Bibliothèque royale de Belgique, Simon Stevin (15481620). L'émergence de la nouvelle science, Turnhout, Brepols, 2004, p. 109-113.

20. M. DöGEN, Architectvra militaris moderna./ Varijs Historijs, tam veteribus quam novis/confirmata, et praecipuis totius Europae mu/nimentis, ad exemplum adductis exornata, Amstelodami, apud Ludovicum Elzevirium, 1647.

21. K. Jordan signale une nouvelle édition française à Amsterdam, en 1658, Bibliographie zur Geschichte des Festungsbaues..., op. cit., p. 69.

22. Le texte a été écrit à Arcetri, près de Florence, sous la surveillance de l'Inquisition, avec interdiction de toute publication. C'est par l'intermé- 
diaire de Fulgensio Micanzio, ami de Galilée, que le manuscrit est transmis à l'éditeur hollandais Elvezier qui le publie en 1638, Hélène VÉRIN, «Galilée et Antoine de Ville, un courrier sur l'idée de matière ", in J. Montesinos et C. Solis (dir.), Largo campo di philosophare, Eurosymposium Galileo 2001, Madrid, Orotavia, 2001, p. 307-322.

23. Nouvelle édition de l'ouvrage en français à Amsterdam en 1668.

24. Le passage du latin aux langues vernaculaires se fait progressivement et plus tardivement pour les ouvrages scientifiques que pour les ouvrages des ingénieurs; P. Bret et E. Moerman notent : «à l'instar des littéraires, les auteurs scientifiques délaissent de plus en plus souvent le latin pour écrire dans la langue de leur nation, objet d'un intérêt croissant qui donnera naissance à la linguistique. Non seulement ils rédigent de moins en moins en latin, mais ils le lisent moins ", puis « de fait, à partir du milieu du XVIII ${ }^{\mathrm{e}}$ siècle, la maîtrise du latin est si peu assurée que la plupart des lecteurs attendent une traduction, condition nécessaire à l'ouverture d'un espace public de la science ", in P. Bret et E. Moerman, "Sciences et arts ", op. cit., p. 597-598.

25. François Blondel, Sa nouvelle manière de fortifier les places, à Paris, chez Nicolas Langlois en 1683; à La Haye par Arnout Leers en 1684 et en 1686; à Londres par Abel Swall, en 1684; puis La Haye par Henry van Bulderen, en 1688. On recense d'autres éditons à Paris, Chez l'auteur et N. Langlois, 1693, et Chez l'auteur, 1699, à Amsterdam, Pierre Mortier, 1699, enfin à La Haye, G. De Voys, 1711.

26. Neue Manier Vestungen zu bauen erfunden durch Monsieur Blondel [...], Nuremberg, Johann Hoffmann, 1686; Novaia manera ukriepleniiu gorodov, trans. I. Zotov, Moscou, Pechatnyi dvor, 1711.

27. A. Manesson-Mallet, Kriegsarbeit oder neuer Festungsbau, trad. allemande de von Filip Zesen, 1672 et trad. hollandaise par von M. Smallegange, Amsterdam, J. Janssonius van Waesberge en J. van Meurs, 1672.

28. L'édition hollandaise peu soignée de 1684 a servi à la traduction russe qui a été réduite au tome premier, livres premier et second, par Ivan Zotov, d'après les annotations de l'exemplaire conservé dans la bibliothèque de Pierre le Grand communiquées par Irina Melevsky à Émilie d'Orgeix qui me les a transmises, ce dont je la remercie.

29. Par exemple, Sir Jonas More, Modern Fortification, or Elements of Military Architecture, Londres, Obadiah Blagrave, 1689, ou William Allingham, maître à l'École de mathématiques de Westminster (Channel Row), qui, en 1702, dans la troisième édition de son Traité, puis en 1722 dans la quatrièmeédition, expose la « Nouvelle méthode de fortification de Vauban ", A Treatise of Military Orders and the Art of Gunnery, etc., Londres, W. Freeman, 1702, p. 187-213.

30. À Amsterdam, Pierre Mortier publie Véritable manière de bien fortifier de M. de Vauban signée par Du Fay et Cambrai en 1702, avec une traduction la même année en espagnol et une version bilingue français et allemand l'année suivante.

31. The New Method of Fortification, as practised by Monsieur de Vauban [...] with an explication of all terms appertaining to that art: made English. [A translation by Abel Swall of "Manière de fortifier de M. de Vauban », by the Chevalier de Cambray. With plates. Printed for Abel Swall, London, 1691 puis Second edition 1693 ; third edition by W. Allingham, 1702. The fifth edition... To which is now added, A treatise of military orders, and the art of gunnery, etc.] The translator's dedication signed : A.S., i. e. Abel Swall.], London, S. \& E. Ballard, 1748.

32. Jacques Ozanam, A Treatise de la fortification, contenant la méthode ancienne et moderne de la construction et la défense des lieux, écrit en français, fait en anglais et modifié [...] par J. T. Desaguliers, avec une annexe concernant cette " manière de fortifier ce qui est vraiment de M. de Vauban ", tiré d'un livre français, publié par l'abbé Du Fay, Oxford, L. Lichfeld, 1711.

33. Abel Boyer, The Draughts of the most remarkable Fortified Towns of Europe in 44 Copper Plates with a Geographical Description of the Said Places And the History of the Sieges [...] A Work very Useful to all Gentlemen and Officers, Londres, Isaac Cleave, 1701.

34. L'enrôlement par Guillaume d'Orange, roi d'Angleterre, des ingénieurs huguenots a largement contribué à la connaissance des plans des placesfortes de Vauban et de ses techniques de fortification.

35. La traduction allemande du livre du chevalier de Cambray, Teutsch-Redender Vauban anjetzo der Teutschen Nation zu lieb in das Hoch-Teutsche gegeben, Mainz, Ludwig Bourgeat, de 1695, connaît un grand succès (rééd. en 1696, 1702, 1707, 1711). La traduction italienne Fortificatione del Signor de Voban [...] transportato dal francese, Parma, Giuseppe Rosetti, 1695, puis 1705, est celle du texte de Du Fay.

36. Memoirs of Monsieur Goulon. Being a treatise on the attack and defence of a place. To which is added, a Journal of the seige of Ath, in the year 1697, under the conduct of Monsieur de Vauban. For the use of the young gentlemen of the Royal Academy at Woolwich, Londres, C. Bathurst, 1745.

37. C. Goulon, Mémoires pour l'attaque et la défense d'une place [...], Wesel, J. van Wesel, 1706; La Haye, Henry van Bulderen, 1706; Amsterdam, D. de la Feuille, 1706; Amsterdam u La Haye, 1711. Trad. allemandes : Bericht von der Nürnberg, J. Hofmann et E. Strecken, 1709; Nürnberg, Peter Conrad 
Monath, 1737 et Peter Monath, 1761, Breslau et Leipzig, D. Pietsch, 1754. Trad. suédoise, Stockholm, H.Ch. Merckell, 1728. L'édition augmentée du " Journal du siège d'Ath » et du "Directeur des fortifications ", La Haye, Paris, P. Gosse et Claude Jombert, 1730, 1736, Francfort 1743, Amsterdam 1750; traductions en allemand Breslau 1754, Amsterdam, 1754 puis 1764.

38. A. Allent, Histoire du corps impérial du Génie, des sièges et des travaux qu'il a dirigés, et des changements que l'attaque, la défense, la construction et l'administration des forteresses ont reçus en France, Paris, Charles-Lavauzelle, 1805, reprint 2002, et le baron de Valazé dans son introduction au Traité de la défense des places par Monsieur le Maréchal de Vauban. Nouvelle édition augmentée des agendas du maréchal sur l'attaque et la défense et de ses notes critiques sur le discours de Deshoulières, relatif à la défense, Paris, Anselin, 1829, ont clairement signalé l'erreur et identifié l'auteur Deshoulières.

39. Baron de Valazé, Traité de la défense des places par Monsieur le Maréchal de Vauban, op. cit., introduction.

40. Mémoire pour servir d'instruction dans la conduite des sièges et dans la défense des places, dressé par M. le maréchal de Vauban et présenté au roi Louis XIV en 1704, Leyde, Jean et Herman Verbeeck, 1740, in $-4^{\circ}$.

41. L'édition de 1740 à Leyde est doublement erronée, puisqu'elle publie dans une première partie le mémoire sur la conduite des sièges de Vauban composé et adressé à Louvois pour son instruction en 1672, et non celui de 1704 dédié au duc de Bourgogne, et dans une deuxième partie l'écrit de Deshoulières en l'attribuant à Vauban.

42. Pour connaître la version initiale du mémoire de Deshoulières, il est aisé de se référer à la publication du Discours de Deshoulières sur la défense, de l'édition du Traité de la défense des places par le baron de Valazé, op. cit.

43. De fait, le Traité sur la défense des places de Vauban ne sera connu qu'en 1769 par une publication parisienne (imparfaite) de Charles-Antoine Jombert, rééd. en 1781 et 1795 . Il faudra attendre 1829 pour qu'une édition conforme au manuscrit original voie le jour, assurée par le baron de Valazé, maréchal de camp du Génie, pour le compte du ministère de la Guerre, à partir du traité conservé au dépôt des fortifications.

44. Philippe BRAgard, " Menno van Coehoorn (1641-1704), contemporain et rival hollandais de Vauban ", in Michèle Virol et Philippe Bragard (éd.), Actes des congrès de Gravelines et d'Arras, Paris, Association Vauban, 2012, p. 103-144.

45. M. van Coehorn, Nieuwe vestingbouw op een natte of lage horisont, Leeuwarden, Rintjes, 1685, rééd. en 1702. Avant même qu'une traduction française ne soit publiée, un officier français installé dans les Provinces-Unies faisait connaître en français la fortification de Coehorn : Louis F. D'AUBIGNÉ DE TIGNÉ, La défense droite, qui est fortification défensive établie sur les principes fixes, et, nouveaux, de monsieur de Coehoorn, Breda, 1705, in- $8^{\circ}$.

46. Abbé Deidien, Le parfait ingénieur, 1742, p. 103-104, cité par P. Bragard, op. cit., p. 119. Les éditions inchangées en français sont nombreuses : 1706, 1711, 1741, 1743.

47. P. BRAGARD, op. cit.

48. À Barcelone, chez Tomas Piferrer, 1769.

49. Irina Gouzevitch, « De la Moscovie à l'Empire russe : le transfert du savoir technique et scientifique et la construction de l'État russe ", SABIX : Bulletin de la Société des Amis de la Bibliothèque de l'École polytechnique, ${ }^{\circ}$ 33, numéro spécial, mai 2003, p. 1-154.

50. Il n'est pas surprenant que L'Architecture militaire de Leonhard C. Sturm, qui a servi d'expert auprès des Russes lors de la prise d'Azov, ait été traduit à Moscou, en 1709, par A.G. Golovkin. Le traité de l'Allemand Rimpler, La manière de Rimpler de fortifier les villes, 1708,1709, connaîtra deux éditions successives en 1708 et 1709 , et surtout l'ouvrage de Menno von Coehorn, Nieuwe vestingbouw, traduit et publié en 1709 puis 1710. Le seul Français à être traduit sera François Blondel, Nouvelle manière de fortifier les places, en 1711.

51. L'identification du texte de du Fay a été faite par Philippe Bragard que je remercie pour cette information. Voltaire signale cette traduction : Histoire de l'Empire de Russie sous Pierre le Grand, in Les cuvres complètes de Voltaire, Oxford, Voltaire foundation, 1999 , t. 47 , p. 795-796. 